\title{
CENSORED QUANTILE REGRESSION WITH VARYING COEFFICIENTS
}

\author{
Guosheng Yin, Donglin Zeng, and Hui Li \\ The University of Hong Kong, The University of North Carolina at Chapel Hill \\ and Beijing Normal University
}

\begin{abstract}
We propose a varying-coefficient quantile regression model for survival data subject to random censoring. Motivated by the work of Yang (I999.9), quantilebased moments are constructed using covariate-weighted empirical cumulative hazard functions. We estimate regression parameters based on the generalized method of moments. The proposed estimators are shown to be consistent and asymptotically normal. We examine the proposed method with finite sample sizes through simulation studies, and illustrate it with a Richter's syndrome study.

Key words and phrases: Generalized method of moments, local polynomial, regression quantiles, semiparametric models, random censoring, survival data.
\end{abstract}

\section{Introduction}

The median survival time is often used as a summary statistic to characterize patient survival. In contrast to the mean survival time, the median is more robust to outliers or extreme observations. The linear regression, also known as the accelerated failure time model, is a mean-based regression approach to covariate analysis. It formulates a linear model between the logarithm of the failure time $T$ and covariates $\mathbf{Z}$ in the form

$$
\log (T)=\boldsymbol{\beta}_{0}^{T} \mathbf{Z}+\varepsilon
$$

If the distribution of the error $\varepsilon$ is not specified, model (메) is semiparametric, for which estimation methods are typically based on the least squares or rank estimators (Buckley and James (1.97.9); Wsiatis (1990); Wei, Ying, and Lin (1990); and Jin et al. (2003)). The mean-based regression model quantifies the central effects of covariates, but may not capture the full distributional impact of covariates with heterogeneous effects. By contrast, when a properly chosen set of quantiles is modeled simultaneously, we can obtain a global assessment of covariate effects (Knenker and Bassett (1978); Koenker (2010.5)). In quantile regression, the model parameters are estimated by minimizing a quantile-based objective function. The corresponding variances are typically estimated through 
resampling methods to avoid nonparametric functional estimation of the error's density function (Parzen, Wei, and Ying (11994); Horowitz (11998); Bilias, Chen and Ying (2000); Jin, Ying, and Weil (2001); and He and Hul (2002)). Yu, Lu, and Stander (2003) provided a general coverage of various applications of quantile regression.

Censored quantile regression, particularly the so-called Tobit model, has been investigated for fixed-censoring data (Powell (1984); Buchinsky and Hahn (11998)). For random censoring cases, Ying, Jung, and Wei (11995) proposed quantile regression for randomly censored failure time data under the assumption of independence between covariates and censoring. Lindgren (11997) studied generalized $L_{1}$ minimization under censored quantile regression. Yang (1999) derived an estimating equation approach to censored median regression based on the covariate-weighted cumulative hazard function. Portnoy (2003) relaxed the independence condition between covariates and censoring times for censored quantile regression by redistributing weights of censored data to the right. Peng and Huang (2018) developed an estimation method for censored quantile regression based on martingale properties and minimization of a sequence of $L_{1}$-type convex functions. Wang and Wang (200.9) proposed redistributing the censored data to the right by using the local Kaplan-Meier estimator. Based on conditional moment inequalities, Khan and Tamer (200.9) further relaxed model assumptions in quantile regression.

On the other hand, varying-coefficient models characterize the trends of covariate effects over time or some exposure variable (Hastie and Tibshiranil ([1993)), while limited research has been conducted in quantile regression with varying coefficients. Yu and Jones (11998) proposed nonparametric regression quantiles using kernel weighted local linear fitting. Honda (20104) studied the local $L_{1}$ estimation with varying coefficients through local polynomial expansions. Kim (2007) proposed spline-based quantile regression and a Rao-score model fit test in contrast to linear quantile regression. Cai and $X_{11}(201018)$ investigated dynamic quantile estimation for time series data. Neocleous and Portnoy (2010) studied partially linear censored quantile regression with B-splines. Qian and Peng (2010) developed censored quantile regression by incorporating partially functional effects.

By extending the work of Yang (1999), we propose a varying-coefficient quantile regression method with randomly censored survival data. We take a local polynomial expansion (Fan and Gijbels (1996)), and incorporate a kernel function to the empirical cumulative hazard function. The quantile-based estimating equations can be viewed as moment conditions in the generalized method of moments (GMM) framework (Hansen (11982); Hansen, Heaton, and Yaron (1096)). In contrast to likelihood-based approaches, the moments of quantile regression 
can be constructed in a relatively straightforward way, and we can combine the available moments and minimize the GMM objective function to estimate regression quantiles.

The rest of this article is organized as follows. In Section 2, we propose the estimation procedure under the censored varying-coefficient quantile regression model. In Section 3, we establish the consistency and asymptotic normality of the parameter estimates. We examine the finite sample properties of the proposed method using simulation studies in Section 4, and illustrate it with a Richter's syndrome study in Section 5. We give concluding remarks in Section 6 and delineate the proofs of the theorems in the supplementary material on the journal's website.

\section{Varying-coefficient Quantile Regression}

Let $T_{i}$ be the failure time, and let $C_{i}$ be the censoring time for the $i$ th subject, $i=1, \ldots, n$. The associated covariates are denoted by a $p$-vector $\mathbf{Z}_{i}$ and a scalar $W_{i}$ (an exposure variable that may interact with $\mathbf{Z}_{i}$ in a nonlinear way). We observe $X_{i}=\min \left(T_{i}, C_{i}\right)$, and the censoring indicator $\Delta_{i}=I\left(T_{i} \leq C_{i}\right)$, where $I(\cdot)$ is the indicator function. We assume that $T_{i}$ is conditionally independent of $C_{i}$ given the covariates, and $\left(X_{i}, \Delta_{i}, \mathbf{Z}_{i}, W_{i}\right)$ are independent and identically distributed (i.i.d.).

Let $q_{\tau}\left(\cdot \mid \mathbf{Z}_{i}, W_{i}\right)$ be the conditional $\tau$-quantile function given covariates $\mathbf{Z}_{i}$ and $W_{i}$, for $0<\tau<1$. To accommodate nonlinear interactions between covariates, we propose the varying-coefficient quantile regression model in the form

$$
q_{\tau}\left(\log \left(T_{i}\right) \mid \mathbf{Z}_{i}, W_{i}\right)=\boldsymbol{\beta}_{\tau}\left(W_{i}\right)^{T} \mathbf{Z}_{i},
$$

where the first component of $\mathbf{Z}_{i}$ is 1 corresponding to the main effect of $W_{i}$. The error term is $\epsilon_{i}=\log \left(T_{i}\right)-\boldsymbol{\beta}_{\tau}\left(W_{i}\right)^{T} \mathbf{Z}_{i}$, so $q_{\tau}\left(\epsilon_{i} \mid \mathbf{Z}_{i}, W_{i}\right)=0$. We assume independence between $\epsilon_{i}$ and $\mathbf{Z}_{i}$, while $\epsilon_{i}$ may depend on $W_{i}$; this allows for possibly heteroscedastic errors to some extent.

Let $\mathcal{W}$ denote the support of the exposure variable $W$. By Taylor's series expansion, for each chosen $w_{0} \in \mathcal{W}$ we have that

$$
\boldsymbol{\beta}_{\tau}(w) \approx \boldsymbol{\beta}_{\tau}\left(w_{0}\right)+\boldsymbol{\beta}_{\tau}^{[1]}\left(w_{0}\right)\left(w-w_{0}\right)+\cdots+\boldsymbol{\beta}_{\tau}^{[r]}\left(w_{0}\right)\left(w-w_{0}\right)^{r},
$$

where

$$
\boldsymbol{\beta}_{\tau}^{[k]}\left(w_{0}\right)=\left.\frac{1}{k !} \frac{\partial^{k} \boldsymbol{\beta}_{\tau}(w)}{\partial w^{k}}\right|_{w_{0}}, \quad k=1, \ldots, r .
$$

We reparameterize with

$$
\begin{aligned}
\boldsymbol{\xi}_{\tau}\left(w_{0}\right) & =\left\{\boldsymbol{\beta}_{\tau}\left(w_{0}\right)^{T}, \boldsymbol{\beta}_{\tau}^{[1]}\left(w_{0}\right)^{T}, \ldots, \boldsymbol{\beta}_{\tau}^{[r]}\left(w_{0}\right)^{T}\right\}^{T}, \\
\mathbf{Z}_{i}^{*} & =\left\{\mathbf{Z}_{i}^{T}, \mathbf{Z}_{i}^{T}\left(W_{i}-w_{0}\right), \ldots, \mathbf{Z}_{i}^{T}\left(W_{i}-w_{0}\right)^{r}\right\}^{T} .
\end{aligned}
$$


Let $K(\cdot)$ be a kernel density function, $h_{n}$ be a bandwidth, and $K_{h_{n}}(\cdot)=$ $K\left(\cdot / h_{n}\right)$. After the local polynomial expansion, we can compute the $\tau$-quantile residuals

$$
e_{i}(\boldsymbol{\xi})=\log \left(X_{i}\right)-\boldsymbol{\xi}_{\tau}\left(w_{0}\right)^{T} \mathbf{Z}_{i}^{*} .
$$

For notational brevity, we drop the dependence of $\boldsymbol{\xi}_{\tau}\left(w_{0}\right)$ on $w_{0}$ and $\tau$ whenever doing so causes no ambiguity. Using the $j$ th covariate as a weight, the local empirical cumulative hazard function is

$$
\widehat{\Lambda}_{j}(t, \boldsymbol{\xi})=\sum_{e_{i}(\boldsymbol{\xi}) \leq t} \frac{K_{h_{n}}\left(W_{i}-w_{0}\right) Z_{i, j}^{*} \Delta_{i}}{\sum_{k=1}^{n} K_{h_{n}}\left(W_{k}-w_{0}\right) Z_{k, j}^{*} I\left(e_{k}(\boldsymbol{\xi}) \geq e_{i}(\boldsymbol{\xi})\right)},
$$

where $Z_{i, j}^{*}$ is the $j$ th component of $\mathbf{Z}_{i}^{*}, j=1, \ldots,(r+1) p$. The $\tau$-quantile estimating equations can be constructed as

$$
\widehat{\Lambda}_{j}(0, \boldsymbol{\xi})=-\log (1-\tau), \quad j=1, \ldots,(r+1) p .
$$

This leads to $(r+1) p$ moment conditions that can be concatenated as

$$
\mathbf{U}_{n}(\boldsymbol{\xi})=n^{-1} \sum_{i=1}^{n} \mathbf{u}_{i}(\boldsymbol{\xi})
$$

where

$$
\mathbf{u}_{i}(\boldsymbol{\xi})=\left(\begin{array}{c}
\frac{n K_{h_{n}}\left(W_{i}-w_{0}\right) Z_{i, 1}^{*} \Delta_{i} I\left(e_{i}(\boldsymbol{\xi}) \leq 0\right)}{\sum_{j=1}^{n} K_{h_{n}}\left(W_{j}-w_{0}\right) Z_{j, 1}^{*} I\left(e_{j}(\boldsymbol{\xi}) \geq e_{i}(\boldsymbol{\xi})\right)}+\log (1-\tau) \\
\vdots \\
n K_{h_{n}}\left(W_{i}-w_{0}\right) Z_{i,(r+1) p}^{*} \Delta_{i} I\left(e_{i}(\boldsymbol{\xi}) \leq 0\right) \\
\frac{\sum_{j=1}^{n} K_{h_{n}}\left(W_{j}-w_{0}\right) Z_{j,(r+1) p}^{*} I\left(e_{j}(\boldsymbol{\xi}) \geq e_{i}(\boldsymbol{\xi})\right)}{}+\log (1-\tau)
\end{array}\right) .
$$

In the GMM framework (Hansen (1982)), $\boldsymbol{\xi}_{\tau}\left(w_{0}\right)$ is estimated by minimizing the weighted quadratic function

$$
Q_{n}(\boldsymbol{\xi})=\mathbf{U}_{n}(\boldsymbol{\xi})^{T} \boldsymbol{\Omega}_{n}(\boldsymbol{\xi})^{-1} \mathbf{U}_{n}(\boldsymbol{\xi}),
$$

where $\boldsymbol{\Omega}_{n}(\boldsymbol{\xi})=n^{-1} \sum_{i=1}^{n} \mathbf{u}_{i}(\boldsymbol{\xi}) \mathbf{u}_{i}(\boldsymbol{\xi})^{T}-\mathbf{U}_{n}(\boldsymbol{\xi}) \mathbf{U}_{n}(\boldsymbol{\xi})^{T}$.

\section{Asymptotic Theories}

Let $\widehat{\boldsymbol{\xi}}\left(w_{0}\right)$ be the minimizer of $Q_{n}(\boldsymbol{\xi})$ in (区.2), and let $\boldsymbol{\xi}_{0}\left(w_{0}\right)$ denote the true parameter. The conditions needed for developing the asymptotic properties of $\widehat{\boldsymbol{\xi}}\left(w_{0}\right)$ are as follows:

(C1) $(\mathbf{Z}, W)$ has a bounded support.

(C2) The joint density of $(T, C, \mathbf{Z}, W)$ is twice-continuously differentiable and is bounded away from zero on its support. 
(C3) $P\left(C \geq c_{0} \mid \mathbf{Z}, W\right)=P\left(C=c_{0} \mid \mathbf{Z}, W\right)>0$, where $c_{0}$ is the study duration.

(C4) $K(\cdot)$ is a symmetric density function and has a finite $(2 r+2)$-moment.

(C5) $w_{0}$ is an interior point of the support of $W, w_{0} \in \mathcal{W}$.

(C6) $\boldsymbol{\beta}_{0}\left(w_{0}\right)$ is $(r+1)$-continuously differentiable at $w_{0}$ with $r \geq 0$.

(C7) $h_{n} \rightarrow 0$, and $n h_{n} \rightarrow \infty$.

(C8) $n h_{n}^{2 r+3}=O(1)$.

Conditions (C1) - (C3) are standard assumptions in the context of survival analysis. The smoothness conditions for $\boldsymbol{\beta}_{0}(\cdot)$ and the kernel function in $(\mathrm{C} 4)-(\mathrm{C} 6)$ allow us to carry out estimation using $r$ th order local polynomials at $w_{0}$. The bandwidth condition $(\mathrm{C} 7)$ controls the variability of the local polynomial estimator, while (C8) implies that the bias of the proposed estimator can be up to $\sqrt{n h_{n}^{2 r+3}}$-order. From the last two conditions, one choice of $h_{n}$ is $n^{-1 /(2 r+3)}$.

Theorem 1. If $(\mathrm{C} 1)-(\mathrm{C} 7)$ hold, $\widehat{\boldsymbol{\xi}}\left(w_{0}\right)$ is a uniformly consistent estimator of $\boldsymbol{\xi}_{0}\left(w_{0}\right)$ for $w_{0} \in \mathcal{W}$.

Set $\mu_{k}=\int u^{k} K(u) d u$ and $\mathbf{H}=\left\{\left(\mu_{k+j}\right)_{(r+1) \times(r+1)} \times \operatorname{Diag}\left(1, h_{n}, \ldots, h_{n}^{r}\right)\right\} \otimes$ $\mathbf{I}_{p \times p}$, where $\left(\mu_{k+j}\right)_{(r+1) \times(r+1)}$ is a $(r+1) \times(r+1)$ dimensional matrix with elements $\mu_{k+j}(k, j=0, \ldots, r)$. Here, $\otimes$ is the Kronecker product, and $\mathbf{I}_{p \times p}$ is the $p$-dimensional identity matrix.

Theorem 2. If $(\mathrm{C} 1)-(\mathrm{C} 8)$ hold, for each interior point $w_{0} \in \mathcal{W}$,

$$
\sqrt{n h_{n}}\left\{\mathbf{H}\left(\widehat{\boldsymbol{\xi}}\left(w_{0}\right)-\boldsymbol{\xi}_{0}\left(w_{0}\right)\right)-h_{n}^{r+1} \mathbf{D}^{-1} \mathbf{b}\right\} \stackrel{\mathcal{D}}{\longrightarrow} N(\mathbf{0}, \boldsymbol{\Sigma})
$$

where $\mathbf{D}, \mathbf{b}$, and $\boldsymbol{\Sigma}$ are given in the supplementary material.

The proofs of the theorems are outlined in the supplementary material; they rely on empirical process theory (van der Vaart and Wellner (1996); Kosorok $(2018))$. As the variance of $\widehat{\boldsymbol{\xi}}\left(w_{0}\right)$ depends on the density of the error, we use the bootstrap procedure to estimate the standard errors of the parameter estimates so as to avoid nonparametric functional estimation.

The first $p$ components of $\boldsymbol{\xi}\left(w_{0}\right)$ correspond to $\boldsymbol{\beta}\left(w_{0}\right)$. Take $\beta_{j}\left(w_{0}\right)$ to be the $j$ th element of $\boldsymbol{\beta}\left(w_{0}\right)$. The optimal bandwidth can be obtained by minimizing the weighted mean squared error

$$
\int\left\{\operatorname{Bias}\left(\hat{\beta}_{j}(w)\right)^{2}+\operatorname{Var}\left(\hat{\beta}_{j}(w)\right)\right\} \Psi(w) d w
$$


where $\Psi(\cdot)$ is a nonnegative and integrable weight function. From Theorem 2, the weighted mean squared error is given by

$$
\int\left\{h_{n}^{2 r+2} \phi_{j}^{2}(w)+\frac{1}{n h_{n}} \sigma_{j j}(w)\right\} \Psi(w) d w
$$

where $\phi_{j}(\cdot)$ is the $j$ th element of the vector $\mathbf{D}^{-1} \mathbf{b}$ and $\sigma_{j j}(\cdot)$ is the $j$ th diagonal element of $\boldsymbol{\Sigma}$. As a result, we can obtain an optimal $h_{n}$ by minimizing the overall mean squared error

$$
\sum_{j=1}^{p} \int\left\{\operatorname{Bias}\left(\hat{\beta}_{j}(w)\right)^{2}+\operatorname{Var}\left(\hat{\beta}_{j}(w)\right)\right\} \Psi(w) d w .
$$

This leads to

$$
h_{n}=n^{-1 /(2 r+3)}\left\{\frac{\sum_{j=1}^{p} \int \sigma_{j j}(w) \Psi(w) d w}{(2 r+2) \sum_{j=1}^{p} \int \phi_{j}^{2}(w) \Psi(w) d w}\right\}^{1 /(2 r+3)} .
$$

In practice, we can use a $K$-fold cross-validation approach to selecting the bandwidth (Hoover et al. ([1998)). We divide the data into $K$ equal-sized subgroups, denoted by $D_{k}, k=1, \ldots, K$. For the data excluding $D_{k}$, we fit the $\tau$ quantile regression model to obtain the parameter estimates $\hat{\boldsymbol{\beta}}_{(-k)}\left(W_{i}\right)$. We then estimate the residual for each subject belonging to $D_{k}, \hat{e}_{i}=\log X_{i}-\widehat{\beta}_{(-k)}\left(W_{i}\right)^{T} \mathbf{Z}_{i}$ for $i \in D_{k}$, and construct the empirical cumulative hazard function based on $\hat{e}_{i}$,

$$
\tilde{\Lambda}_{k}(0)=\sum_{\hat{e}_{i} \leq 0, i \in D_{k}} \frac{\Delta_{i}}{\sum_{j \in D_{k}} I\left(\hat{e}_{j} \geq \hat{e}_{i}\right)} .
$$

We find the optimal bandwidth by minimizing $\sum_{k=1}^{K}\left|\tilde{\Lambda}_{k}(0)+\log (1-\tau)\right|$.

\section{Simulation Studies}

\subsection{Homogeneous error}

To examine the finite sample property of the proposed method, we conducted extensive simulation studies. We first considered a model with homogeneous errors,

$$
\log (T)=\beta_{0}(W)+\beta_{1}(W) Z_{1}+\beta_{2}(W) Z_{2}+\epsilon,
$$

where $\beta_{0}(W)=0.5, \beta_{1}(W)=W^{2}$, and $\beta_{2}(W)=\cos (3 W)$. The covariates $W, Z_{1}$, and $Z_{2}$ were $\operatorname{Unif}(-1,1), \operatorname{Unif}(0,1)$, and $N(0,1)$, respectively; the error $\epsilon$ was $N(0,1)$. Censoring times were independently generated from a uniform distribution to yield a censoring rate of $25 \%$. We partitioned the range of $W$, $[-1,1]$, into 20 equal intervals to evaluate the coefficient functions. As the GMM 
Table 1. Estimation of the regression coefficients and the corresponding derivatives under model (4.D) with $25 \%$ censoring.

\begin{tabular}{|c|c|c|c|c|c|c|c|c|c|c|c|}
\hline$w_{0}$ & $h_{n}$ & True & $\widehat{\beta}\left(w_{0}\right)$ & SD & SE & $\mathrm{CP}(\%)$ & True & $\widehat{\beta}^{\prime}\left(w_{0}\right)$ & SD & SE & $\mathrm{CP}(\%)$ \\
\hline \multirow{3}{*}{-0.5} & \multirow[b]{2}{*}{0.06} & \multicolumn{5}{|c|}{$\beta_{0}(w)=0.5$} & \multicolumn{5}{|c|}{$\beta_{0}^{\prime}(w)=0$} \\
\hline & & \multirow[t]{2}{*}{0.5} & 0.592 & 0.464 & 0.520 & 96.8 & \multirow[t]{2}{*}{0} & 0.113 & 2.208 & 2.461 & 96.8 \\
\hline & 0.08 & & 0.547 & 0.391 & 0.454 & 97.2 & & -0.008 & 1.595 & 1.792 & 97.2 \\
\hline \multirow[t]{2}{*}{0} & 0.06 & 0.5 & 0.534 & 0.439 & 0.506 & 97.2 & \multirow[t]{2}{*}{0} & 0.270 & 1.886 & 2.205 & 97.0 \\
\hline & 0.08 & \multirow{3}{*}{0.5} & 0.521 & 0.389 & 0.432 & 96.0 & & 0.037 & 1.403 & 1.578 & 96.6 \\
\hline \multirow[t]{3}{*}{0.5} & 0.06 & & 0.523 & 0.498 & 0.538 & 96.6 & \multirow{2}{*}{0} & 0.414 & 1.945 & 2.323 & 97.4 \\
\hline & 0.08 & & 0.483 & 0.419 & 0.455 & 96.2 & & 0.208 & 1.350 & 1.616 & 98.0 \\
\hline & & \multicolumn{5}{|c|}{$\beta_{1}(w)=w^{2}$} & \multicolumn{5}{|c|}{$\beta_{1}^{\prime}(w)=2 w$} \\
\hline \multirow[t]{2}{*}{-0.5} & 0.06 & 0.25 & 0.262 & 0.800 & 0.886 & 98.0 & \multirow[t]{2}{*}{-1.0} & -0.232 & 3.394 & 3.730 & 97.4 \\
\hline & 0.08 & & 0.334 & 0.706 & 0.780 & 97. & & -0.075 & 2.585 & 2.869 & 96.6 \\
\hline \multirow[t]{2}{*}{0} & 0.06 & 0 & 0.162 & 0.780 & 0.872 & 97 . & \multirow[t]{2}{*}{0} & 0.257 & 2.926 & 2.331 & 97.2 \\
\hline & 0.08 & & 0.132 & 0.683 & 0.754 & 97. & & 0.475 & 2.288 & 2.481 & 96.2 \\
\hline \multirow[t]{3}{*}{0.5} & 0.06 & 0.25 & 0.434 & 0.934 & 0.913 & 97.8 & \multirow[t]{2}{*}{1.0} & 0.748 & 3.088 & 3.431 & 96.4 \\
\hline & \multirow[t]{2}{*}{0.08} & & 0.485 & 0.818 & 0.782 & 98.8 & & 0.954 & 2.094 & 2.507 & 97.6 \\
\hline & & \multicolumn{5}{|c|}{$\beta_{2}(w)=\cos (3 w)$} & \multicolumn{5}{|c|}{$\beta_{2}^{\prime}(w)=-3 \sin (3 w)$} \\
\hline \multirow[t]{2}{*}{-0.5} & 0.06 & 0.071 & 0.141 & 0.477 & 0.506 & 94.8 & \multirow[t]{2}{*}{$\begin{array}{l}2.992 \\
\end{array}$} & 2.530 & 1.576 & 1.816 & 96.4 \\
\hline & 0.08 & & 0.210 & 0.515 & 0.464 & 95.4 & & 2.465 & 1.012 & 1.234 & 96.2 \\
\hline \multirow[t]{2}{*}{0} & 0.06 & 1.000 & 0.917 & 0.380 & 0.460 & 97.0 & \multirow[t]{2}{*}{0} & 0.353 & 1.545 & 1.826 & 98.2 \\
\hline & 0.08 & & 0.912 & 0.385 & 0.420 & 95.2 & & 0.274 & 1.030 & 1.197 & 97.2 \\
\hline \multirow[t]{2}{*}{0.5} & 0.06 & 0.071 & 0.171 & 0.561 & 0.521 & 91.6 & \multirow[t]{2}{*}{-2.992} & -2.059 & 1.640 & 2.012 & 95.4 \\
\hline & 0.08 & & 0.130 & 0.474 & 0.485 & 95.6 & & -2.095 & 1.005 & 1.282 & 93.4 \\
\hline
\end{tabular}

$\mathrm{SD}$ is the standard deviation, SE is the estimated standard error using the bootstrap method averaged over 500 simulations, $\mathrm{CP}(\%)$ is the $95 \%$ confidence interval coverage probability.

objective function in ([2.2) is complicated and highly nonlinear with respect to the parameters, we applied the Nelder and Mead (1965) simplex algorithm to minimize the quadratic function. We took a local linear expansion with $r=1$, chose the Gaussian kernel function and explored the bandwidths $h_{n}=0.06$ and 0.08. The sample size was $n=200$, and we took 400 bootstrap samples for variance estimation. For each configuration, we replicated 500 simulations.

Table 1 summarizes the estimation results for $w_{0}=-0.5,0$, and 0.5 . We present the average of the varying-coefficient estimates, the standard deviation (SD), the average of the estimated standard errors (SE) based on the bootstrap method, and the coverage probability of the $95 \%$ confidence interval (CP\%). We also provide the estimates for the corresponding derivatives of the varying coefficients. One can see that the estimation bias is small, the bootstrap variance estimate provides a fairly good approximation to the variability of the estimators, and the coverage probability reasonably matches the nominal level. Under 
(a) Estimated curves of $\beta_{0}(w)=0.5$

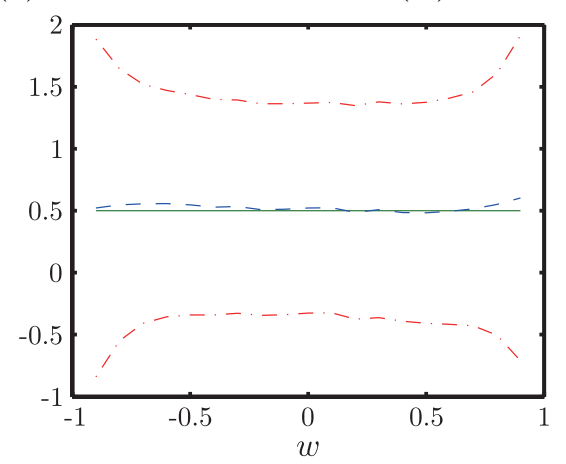

(c) Estimated curves of $\beta_{1}(w)=w^{2}$

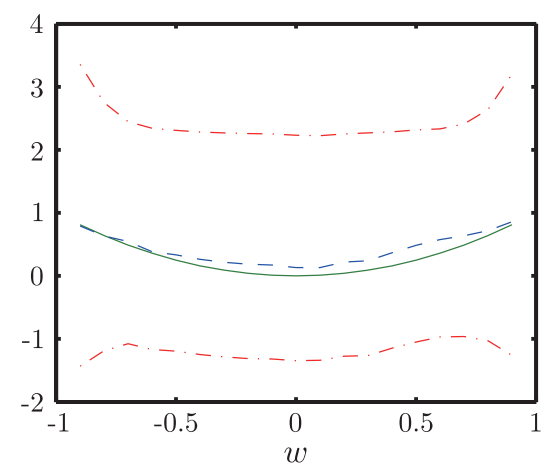

(e) Estimated curves of $\beta_{2}(w)=\cos (3 w)$



(b) Estimated curves of $\beta_{0}^{\prime}(w)=0$

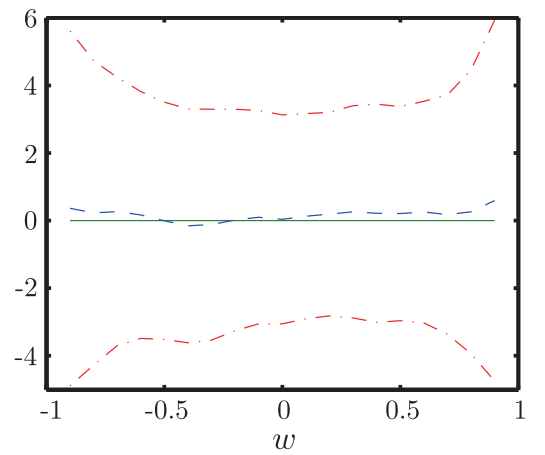

(d) Estimated curves of $\beta_{1}^{\prime}(w)=2 w$

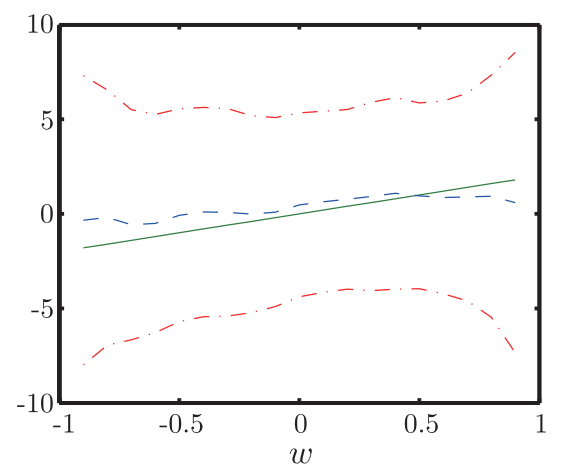

(f) Estimated curves of $\beta_{2}^{\prime}(w)=-3 \sin (3 w)$

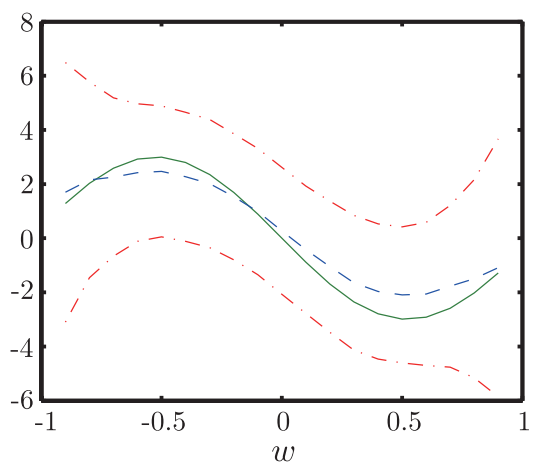

Figure 1. Estimation results under model (4. 1) with $n=200,25 \%$ censoring and $h_{n}=0.08$. (a) $-(\mathrm{f})$ : solid lines are the true functions and dashed lines are the estimates of varying coefficients coupled with the pointwise $95 \%$ confidence intervals.

different bandwidths, the simulation results were similar, indicating to a certain extent the robustness of the method with respect to the bandwidth. Figure 1 shows both the estimated varying-coefficient functions and their derivatives with 
Table 2. Estimation of the regression coefficients under model (4.2) with varying and constant coefficients.

\begin{tabular}{|c|c|c|c|c|c|c|c|c|c|c|c|c|c|c|}
\hline \multirow[b]{2}{*}{$n$} & \multirow[b]{2}{*}{$c \%$} & \multirow[b]{2}{*}{$w_{0}$} & \multicolumn{4}{|c|}{$\beta_{0}(w)=w^{2}$} & \multicolumn{4}{|c|}{$\beta_{1}(w)=\sin (3 w)$} & \multicolumn{4}{|c|}{$\gamma=0.5$} \\
\hline & & & Bias & SD & $\mathrm{SE}$ & $\mathrm{CP}(\%)$ & Bias & $\mathrm{SD}$ & $\mathrm{SE}$ & $\mathrm{CP}(\%)$ & Bias & $\mathrm{SD}$ & $\mathrm{SE}$ & $\mathrm{CP}(\%)$ \\
\hline \multirow[t]{9}{*}{200} & 0 & -0.7 & -0.024 & 0.182 & 0.226 & 97.0 & 0.128 & 0.268 & 0.333 & 95.4 & 0.004 & 0.089 & 0.093 & 96.2 \\
\hline & & 0 & -0.060 & 0.161 & 0.180 & 94.0 & -0.008 & 0.253 & 0.276 & 94.0 & & & & \\
\hline & & 0.7 & 0.022 & 0.189 & 0.232 & 97.0 & -0.100 & 0.307 & 0.314 & 94.4 & & & & \\
\hline & 20 & -0.7 & -0.019 & 0.193 & 0.254 & 98.4 & 0.127 & 0.307 & 0.368 & 96.6 & 0.023 & 0.100 & 0.103 & 91.6 \\
\hline & & 0 & 0.051 & 0.165 & 0.196 & 96.6 & -0.011 & 0.262 & 0.305 & 97.6 & & & & \\
\hline & & 0.7 & 0.050 & 0.239 & 0.285 & 97.2 & -0.144 & 0.343 & 0.387 & 95.0 & & & & \\
\hline & 40 & -0.7 & 0.020 & 0.252 & 0.299 & 97.8 & 0.115 & 0.399 & 0.440 & 96.0 & 0.034 & 0.113 & 0.126 & 96.0 \\
\hline & & 0 & 0.072 & 0.186 & 0.234 & 96.4 & -0.025 & 0.284 & 0.347 & 97.4 & & & & \\
\hline & & 0.7 & 0.121 & 0.318 & 0.405 & 96.6 & -0.078 & 0.438 & 0.498 & 96.6 & & & & \\
\hline \multirow[t]{9}{*}{400} & 0 & -0.7 & 0.034 & 0.131 & 0.144 & 95.6 & 0.086 & 0.198 & 0.225 & 95.4 & 0.001 & 0.059 & 0.062 & 95.8 \\
\hline & & 0 & 0.035 & 0.123 & 0.137 & 94.8 & 0.009 & 0.182 & 0.213 & 97.4 & & & & \\
\hline & & 0.7 & 0.021 & 0.130 & 0.147 & 96.4 & -0.078 & 0.204 & 0.226 & 94.4 & & & & \\
\hline & 20 & -0.7 & 0.034 & 0.141 & 0.162 & 97.2 & 0.082 & 0.223 & 0.252 & 95.6 & -0.001 & 0.061 & 0.069 & 96.8 \\
\hline & & 0 & 0.039 & 0.131 & 0.147 & 95.4 & 0.008 & 0.202 & 0.231 & 97.4 & & & & \\
\hline & & 0.7 & 0.033 & 0.152 & 0.191 & 98.2 & -0.080 & 0.243 & 0.280 & 96.6 & & & & \\
\hline & 40 & -0.7 & 0.026 & 0.157 & 0.197 & 98.2 & 0.087 & 0.248 & 0.296 & 95.4 & 0.010 & 0.082 & 0.087 & 96.2 \\
\hline & & 0 & 0.048 & 0.143 & 0.172 & 96.4 & -0.001 & 0.231 & 0.265 & 96.8 & & & & \\
\hline & & 0.7 & 0.134 & 0.342 & 0.353 & 93.4 & -0.068 & 0.330 & 0.390 & 97.8 & & & & \\
\hline
\end{tabular}

$\mathrm{SD}$ is the standard deviation, SE is the estimated standard error using the bootstrap method averaged over 500 simulations, $\mathrm{CP}(\%)$ is the $95 \%$ confidence interval coverage probability.

a bandwidth of $h_{n}=0.08$ coupled with the $95 \%$ confidence intervals. The estimated curves match reasonably with the true functions, while the estimates for the derivatives of the varying coefficients are generally not as good as those for the functions themselves.

To acknowledge that some covariate effects are varying while others are constant, we took

$$
\log (T)=\beta_{0}(W)+\beta_{1}(W) Z_{1}+\gamma Z_{2}+\epsilon,
$$

where $\beta_{0}(W)=W^{2}, \beta_{1}(W)=\sin (3 W)$, and $\gamma=0.5$. The covariate $W$ was $\operatorname{Unif}(-1,1), Z_{1}$ was $\operatorname{Unif}(0,1), Z_{2}$ was Bernoulli(0.5), and the error $\epsilon$ was $N(0,0.25)$. We took the sample sizes $n=200$ and 400 , and the censoring rates $c \%=0,20 \%$, and $40 \%$. A simple way to estimate the constant coefficient $\gamma$ is to first estimate $\gamma\left(w_{0}\right)$, and then take an average over all the chosen $w_{0}$. Table 2 shows that the estimates are quite accurate in general when the model involves both varying and constant coefficients. The bias is small, the standard errors reasonably characterize the variation of the estimates, and the coverage probabilities are around $95 \%$.

For comparison, we also implemented the B-spline method in Neocleous and Portnoy (2010), for which we chose three knots corresponding to the $25 \%, 50 \%$ 
Table 3. Comparison of the proposed method with the B-spline method of Neocleous and Portnoy (200.9) in terms of mean squared errors $\left(\times 10^{-2}\right)$ with a censoring rate of $20 \%$, and $h_{n}=0.06,0.10$, and 0.14 .

\begin{tabular}{|c|c|c|c|c|c|}
\hline \multirow[b]{2}{*}{ Kernel $\left(h_{n}\right)$} & \multicolumn{2}{|c|}{ MSE } & \multirow[b]{2}{*}{ B-spline } & \multicolumn{2}{|c|}{ MSE } \\
\hline & $\beta_{0}\left(w_{0}\right)$ & $\beta_{1}\left(w_{0}\right)$ & & $\beta_{0}\left(w_{0}\right)$ & $\beta_{1}\left(w_{0}\right)$ \\
\hline 0.06 & 3.806 & 3.615 & Linear & 3.714 & 10.862 \\
\hline 0.10 & 9.380 & 3.151 & Quadratic & 4.541 & 14.199 \\
\hline 0.14 & 22.875 & 4.873 & Cubic & 5.267 & 17.399 \\
\hline
\end{tabular}

and $75 \%$ quantiles and explored piecewise linear, quadratic, and cubic spline terms. Table 3 shows that the mean squared errors are comparable between the two methods.

\subsection{Heteroscedastic error}

As quantile regression is known to be most suitable for heteroscedastic errors, we also examined heterogeneity induced by covariate-dependent errors. We simulated failure times from the model

$$
\log (T)=\beta_{0}(W)+\beta_{1}(W) Z+\epsilon,
$$

where $\beta_{0}(W)=2 W$ and $\beta_{1}(W)=\sin (3 W)$. We generated $W$ as $N(0,1), Z$ as Bernoulli(0.5), and the error $\epsilon$ as $W \times N(0,1)$ for $Z=1$ and as $W \times N(0,0.7)$ for $Z=0$. Censoring times were independently uniform and yielded an approximate censoring rate of $20 \%$. We partitioned the range of $W$ into 18 equal intervals to locally evaluate the coefficients at each chosen $w_{0}$. We took a local linear expansion with $r=1$, chose the Gaussian kernel function and considered the bandwidths $h_{n}=0.16,0.18$, and 0.20 . We took the sample size $n=200$ and, for each configuration, we replicated 500 simulations. To handle heteroscedastic errors, we stratified the estimating equation by $Z$, and minimized $Q_{n}(\boldsymbol{\xi} \mid Z=0)$ and $Q_{n}(\boldsymbol{\xi} \mid Z=1)$, respectively. From the simulation results summarized in Table 4 , one can see that the biases of the estimates are small, the standard errors are close to the standard deviations, and the coverage probabilities are reasonable. For $h_{n}=0.18$, we exhibit the estimated curves for $\tau=0.5$ in Figure 2, and those for $\tau=0.25$ in Figure 3. Under (4.3), the computing time using I5-2430M CPU (2.40GHz and 8GB RAM) with FORTRAN PowerStation 4.0 was approximately 1 minute per simulation.

\section{Example}

We applied the proposed model to a data set from a leukemia study conducted at M. D. Anderson Cancer Center (Tsimberidou et al. (2006)). The study involved 130 evaluable patients, diagnosed with Richter's syndrome via 
(a) Estimated curves of $\beta_{0}(w)$

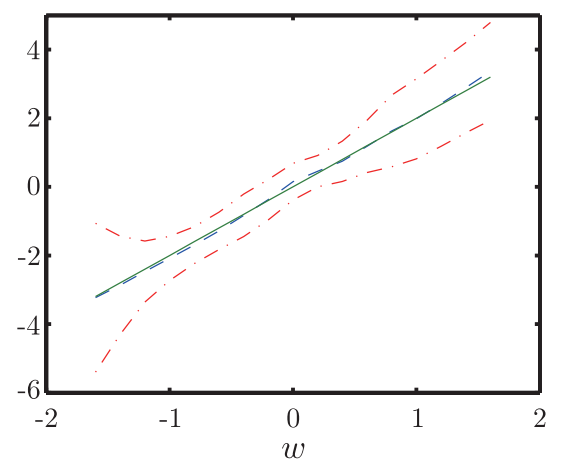

(b) Estimated curves of $\beta_{1}(w)$

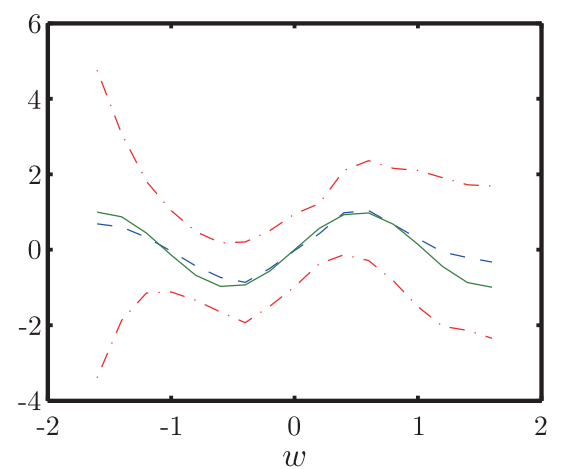

Figure 2. Estimation results under model (4.3) with $n=200, \tau=0.5,20 \%$ censoring and $h_{n}=0.18$. (a)-(b): solid lines are the true functions and dashed lines are the estimates of the varying coefficients coupled with the pointwise $95 \%$ confidence intervals.

(a) Estimated curves of $\beta_{0}(w)$

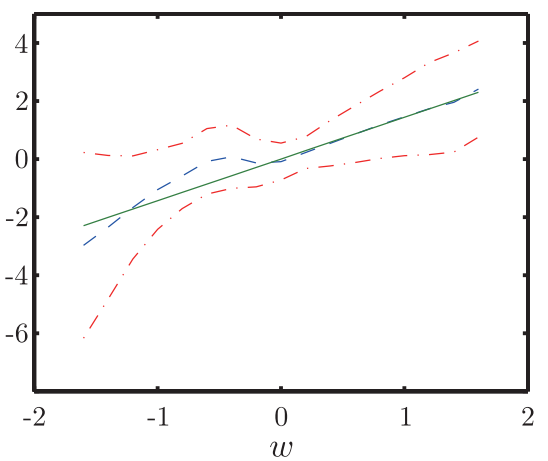

(b) Estimated curves of $\beta_{1}(w)$

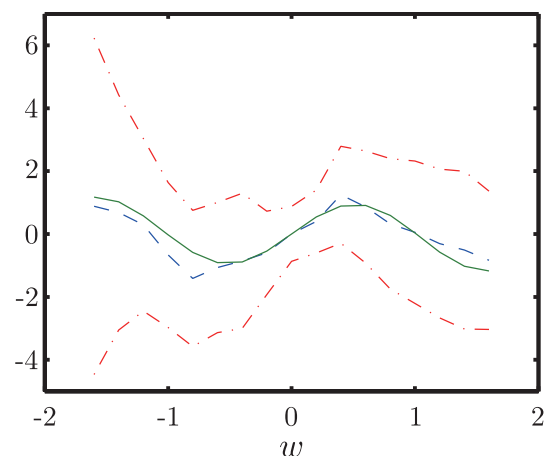

Figure 3. Estimation results under model (4.3) with $n=200, \tau=0.25,20 \%$ censoring and $h_{n}=0.18$. (a)-(b): solid lines are the true functions and dashed lines are the estimates of the varying coefficients coupled with the pointwise $95 \%$ confidence intervals.

biopsy or fine-needle aspiration, who had been examined between January 1975 and June 2005. Richter's syndrome (RS) is a rare and aggressive type of acute adult leukemia that often results from a transformation of chronic lymphocytic leukemia into diffuse large cell lymphoma; it is usually fatal within a short period of time. In this study, patients were treated by chemoimmunotherapy with rituximab or chemotherapy alone. Figure 4 exhibits the Kaplan-Meier survival curves for the 130 patients with RS. We can see a sharp change-point in the survival curve around two years of follow-up, which would typically cause the violation of the usual proportional hazards assumption ( $\operatorname{Cox}($ (1972)). The censoring rate of the data was approximately $12 \%$. 
Table 4. Estimation of the regression coefficients under model (4.3) with a censoring rate of $20 \%$, and $h_{n}=0.16,0.18$, and 0.2 .

\begin{tabular}{|c|c|c|c|c|c|c|c|c|c|c|c|}
\hline \multirow[b]{2}{*}{$w_{0}$} & \multirow[b]{2}{*}{$h$} & \multicolumn{5}{|c|}{$\beta_{0}(w)=2 w$} & \multicolumn{5}{|c|}{$\beta_{1}(w)=\sin (3 w)$} \\
\hline & & True & $\widehat{\beta}_{0}\left(w_{0}\right)$ & SD & $\mathrm{SE}$ & $\mathrm{CP}(\%)$ & True & $\widehat{\beta}_{1}\left(w_{0}\right)$ & SD & $\mathrm{SE}$ & $\mathrm{CP}(\%)$ \\
\hline \multirow[t]{3}{*}{-1.2} & 0.16 & -2.4 & -2.459 & 0.471 & 0.453 & 96.6 & 0.443 & 0.307 & 0.484 & 0.714 & 98.6 \\
\hline & 0.18 & & -2.463 & 0.339 & 0.479 & 96.8 & & 0.284 & 0.528 & 0.744 & 99.4 \\
\hline & 0.20 & & -2.517 & 0.464 & 0.483 & 95.4 & & 0.285 & 0.498 & 0.792 & 98.4 \\
\hline \multirow[t]{3}{*}{-0.8} & 0.16 & -1.6 & -1.660 & 0.402 & 0.259 & 97.0 & -0.675 & -0.487 & 0.361 & 0.500 & 97.8 \\
\hline & 0.18 & & -1.682 & 0.287 & 0.283 & 95.2 & & -0.462 & 0.367 & 0.519 & 97.2 \\
\hline & 0.20 & & -1.700 & 0.428 & 0.314 & 93.6 & & -0.419 & 0.460 & 0.555 & 96.4 \\
\hline \multirow[t]{3}{*}{-0.4} & 0.16 & -0.8 & -0.811 & 0.315 & 0.303 & 97.6 & -0.932 & -0.854 & 0.405 & 0.485 & 98.2 \\
\hline & 0.18 & & -0.811 & 0.411 & 0.319 & 97.2 & & -0.885 & 0.527 & 0.551 & 98.4 \\
\hline & 0.20 & & -0.833 & 0.423 & 0.327 & 96.6 & & -0.900 & 0.476 & 0.568 & 97.8 \\
\hline \multirow[t]{3}{*}{0} & 0.16 & 0 & 0.180 & 0.501 & 0.278 & 96.0 & 0 & -0.058 & 0.722 & 0.473 & 99.2 \\
\hline & 0.18 & & 0.184 & 0.532 & 0.265 & 94.4 & & 0.023 & 0.672 & 0.471 & 97.0 \\
\hline & 0.20 & & 0.158 & 0.554 & 0.240 & 95.0 & & -0.012 & 0.639 & 0.454 & 97.8 \\
\hline \multirow[t]{3}{*}{0.4} & 0.16 & 0.8 & 0.779 & 0.323 & 0.302 & 98.0 & 0.932 & 0.937 & 0.506 & 0.557 & 98.2 \\
\hline & 0.18 & & 0.775 & 0.336 & 0.317 & 97.2 & & 0.996 & 0.639 & 0.606 & 98.4 \\
\hline & 0.20 & & 0.755 & 0.339 & 0.298 & 97.2 & & 1.016 & 0.632 & 0.613 & 97.6 \\
\hline \multirow[t]{3}{*}{0.8} & 0.16 & 1.6 & 1.612 & 0.504 & 0.467 & 97.6 & 0.675 & 0.776 & 0.767 & 0.771 & 97.8 \\
\hline & 0.18 & & 1.646 & 0.734 & 0.505 & 95.8 & & 0.700 & 0.667 & 0.774 & 98.4 \\
\hline & 0.20 & & 1.643 & 0.766 & 0.625 & 97.0 & & 0.711 & 0.656 & 0.805 & 97.4 \\
\hline \multirow[t]{3}{*}{1.2} & 0.16 & 2.4 & 2.502 & 0.612 & 0.674 & 96.2 & -0.443 & -0.016 & 0.804 & 0.956 & 92.2 \\
\hline & 0.18 & & 2.438 & 0.492 & 0.637 & 96.8 & & -0.017 & 0.936 & 0.988 & 93.8 \\
\hline & 0.20 & & 2.409 & 0.448 & 0.644 & 97.4 & & -0.057 & 0.795 & 0.972 & 93.4 \\
\hline
\end{tabular}

SD is the standard deviation, SE is the estimated standard error using the bootstrap method averaged over 500 simulations, $\mathrm{CP}(\%)$ is the $95 \%$ confidence interval coverage probability.

In the varying-coefficient quantile regression model, we included three covariates: treatment ( 1 if using chemotherapy alone, and 0 if using chemoimmunotherapy with rituximab), age (ranging from 29 to 77 years with a median of 60 years), and sex ( 1 if male, and 0 if female). We were interested in characterizing the nonlinear interactions between patient age and other covariates and how they affected the quantiles of patient survival times. With $\tau=0.25,0.5$, and 0.75 , we fit the proposed varying-coefficient quantile regression model

$$
q_{\tau}(\log (T) \mid \mathbf{Z}, W)=\beta_{0}(W)+\beta_{1}(W) Z_{\text {treatment }}+\beta_{2}(W) Z_{\text {sex }},
$$

where $W$ is the logarithm of patient age. In this analysis, we divided the data into five groups of 26 observations each. Based on the five-fold cross-validation procedure described earlier, the bandwidth $h_{n}=0.19$ appeared to be a reasonable choice. We partitioned the range of $W$ into 20 equal intervals. The estimated coefficient functions and the corresponding $95 \%$ confidence intervals 


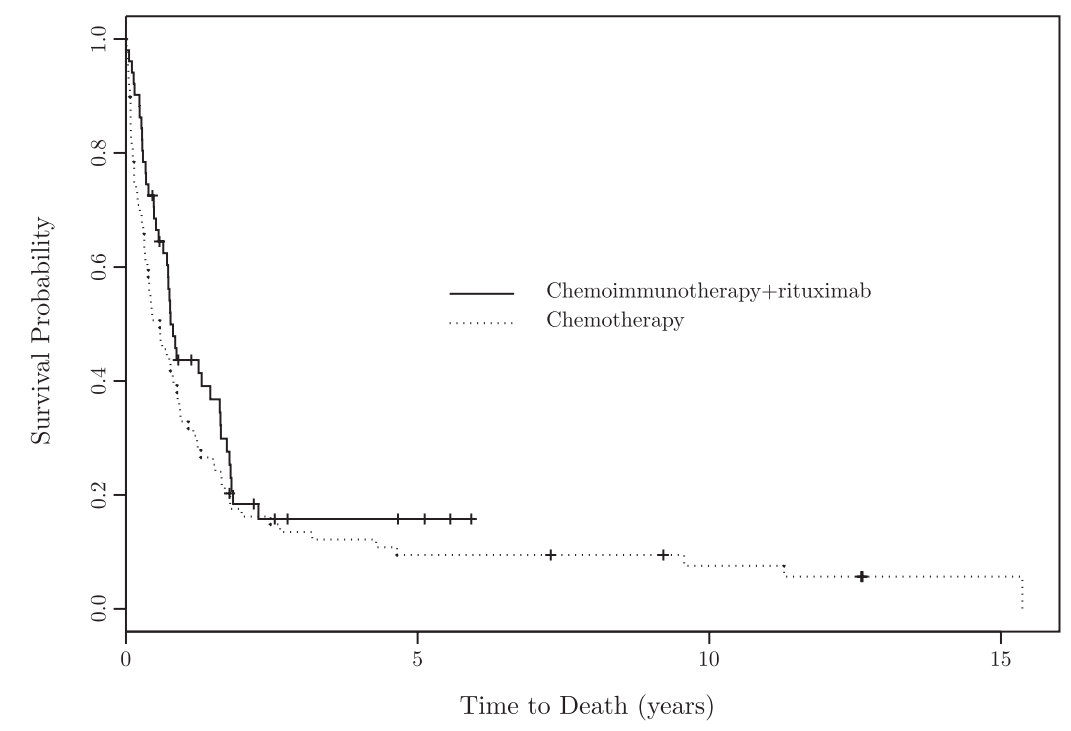

Figure 4. Estimated Kaplan-Meier survival curves stratified by treatment for the Richter's syndrome data.

are given in Figure 5. In the median regression, there was a trend that treatment with chemoimmunotherapy and rituximab improved patient survival while such difference diminished as patient age increased. This trend appeared to be similar for the other two conditional quantiles as well. With regard to the covariate effect of patient sex, we did not find any quantile difference between male and female patients for $\tau=0.5$. For $\tau=0.25$, it appeared that younger male patients had better survival than younger female patients, while older male patients had worse survival than older female patients. For $\tau=0.75$, male patients seemed to have better survival than female regardless of their ages. Generally, all these findings are not statistically significant, they only exhibit some trends in patients' survival with respect to different covariates.

\section{Discussion}

We have proposed censored quantile regression with varying coefficients by adopting the estimation method of Yang (1.999) due to the simplicity of constructing the covariate-weighted cumulative hazard function. By taking the local polynomial expansion of varying-coefficient functions, we construct the kernelbased moments such that the estimation procedure can be naturally integrated with the GMM. Although the GMM involves complicated nonlinear estimating equations, our numerical studies have shown that the estimators perform reasonably well with finite sample sizes. The proposed method allows the error to depend on the exposure variable $W$, and thus can handle heteroscedastic errors to 

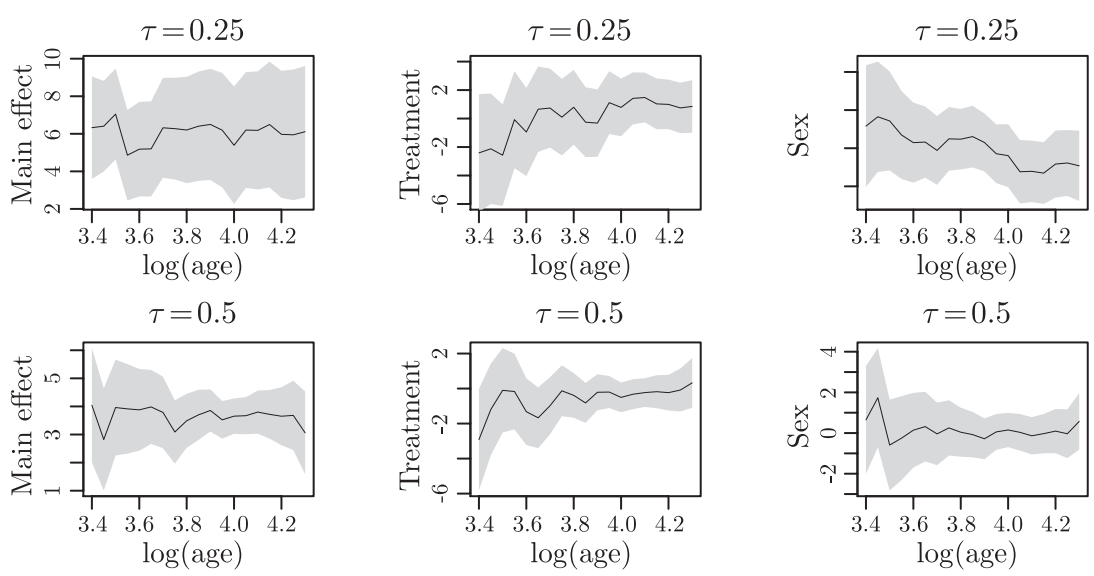

$\tau=0.75$
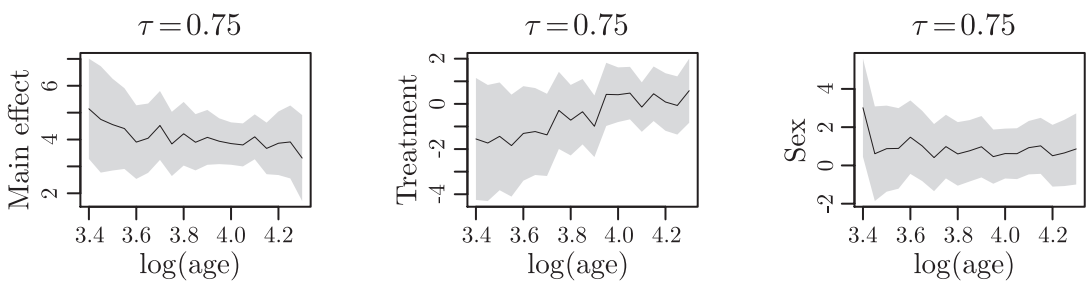

Figure 5. Analysis of the Richter's syndrome data based on the proposed varying-coefficient quantile regression. The curve is the estimated varying coefficients and the shaded area is the pointwise $95 \%$ confidence interval.

some extent. We may also allow the error to depend on covariate $\mathbf{Z}$ by stratification for discrete $\mathbf{Z}$, or by local kernel estimation for continuous $\mathbf{Z}$. Nevertheless, if the dimension of $\mathbf{Z}$ is high, estimation can be challenging. The partially linear quantile regression model in Neocleous and Portnoy (200.9) can accommodate general heteroscedasticty, while to carry out median regression analysis, all the regression quantiles below the median must be calculated first.

In censored quantile regression, it is known that the estimation of upper quantiles may not be stable due to identifiability issues. In general, $\tau$ should be smaller than $\inf _{\mathbf{z}, w_{0}} P\left(T \leq c_{0} \mid \mathbf{Z}=\mathbf{z}, W=w_{0}\right)$, where $c_{0}$ is the study end time, so that we would have data to estimate the $\tau$-quantile. For varying-coefficient models, it is important to determine whether covariate effects are varying or constant over the exposure variable $W$. Toward this goal, some model goodness-of-fit procedures might be considered (He and Zhu (2003)), and automatic discovery procedures (Zhang, Cheng, and Liu (2017)) also warrant further research.

\section{Acknowledgement}

We would like to thank the two anonymous referees, an associate editor, and Editor Qiwei Yao for many insightful suggestions that strengthened the work 
immensely. We also thank Professor Don Ylvisaker for his enormous editorial help. This work is partially supported by a grant (Grant No. 784010) from the Research Grants Council of Hong Kong (G. Yin), and a grant (Grant No. 11201031) from National Natural Science Foundation of China (H. Li).

\section{References}

Bilias, Y., Chen, S. and Ying, Z. (2000). Simple resampling methods for censored regression quantiles. J. Econometrics 99, 373-386.

Buchinsky, M. and Hahn, J. Y. (1998). An alternative estimator for censored quantile regression. Econometrica 66, 653-671.

Buckley, J. and James, I. (1979). Linear regression with censored data. Biometrika 66, 429-436.

Cai, Z. and Xu, X. (2008). Nonparametric quantile estimations for dynamic smooth coefficient models. J. Amer. Statist. Assoc., 1595-1608.

Cox, D. R. (1972). Regression models and life tables (with discussion). J. Roy. Statist. Soc. Ser. B 34, 187-220

Fan, J. and Gijbels, I. (1996). Local Polynomial Modelling and Its Applications. Chapman \& Hall, London.

Hansen, L. P. (1982). Large sample properties of generalized method of moments estimators. Econometrica 50, 1029-1054.

Hansen, L. P., Heaton, J. and Yaron, A. (1996). Finite-sample properties of some alternative GMM estimators. J. Bus. Econom. Statist. 14, 262-280.

Hastie, T. and Tibshirani, R. (1993). Varying-coefficient models. J. Roy. Statist. Soc. Ser. B 55, 757-796.

He, X. and Hu, F. (2002). Markov chain marginal bootstrap. J. Amer. Statist. Assoc. 97, 783795.

He, X. and Zhu, L.-X. (2003). A lack-of-fit test for quantile regression. J. Amer. Statist. Assoc. 98, 1013-1022.

Honda, T. (2004). Quantile regression in varying coefficient models. J. Statist. Plann. Inference 121, 113-125.

Hoover, D. R., Rice, J. A., Wu, C. O. and Yang, L. P. (1998). Nonparametric smoothing estimates of time-varying coefficient models with longitudinal data. Biometrika 85, 809822 .

Horowitz, J. (1998). Bootstrap methods for median regression model. Econometrica 66, 13271352.

Jin, Z., Lin, D. Y., Wei, L. J. and Ying, Z. (2003). Rank-based inference for the accelerated failure time model. Biometrika 90, 341-353.

Jin, Z., Ying, Z. and Wei, L. J. (2001). A simple resampling method by perturbing the minimand. Biometrika 88, 381-390.

Khan, S. and Tamer, E. (2009). Inference on endogenously censored regression models using conditional moment inequalities. J. Econometrics 152, 104-119.

Kim, M-O. (2007). Quantile regression with varying coefficients. Ann. Statist. 35, 92-108.

Koenker, R. (2005). Quantile Regression. Cambridge University Press.

Koenker, R. and Bassett, G. J. (1978). Regression quantiles. Econometrica 46, 33-50.

Kosorok, M. R. (2008). Introduction to Empirical Processes and Semiparametric Inference. Springer, New York. 
Lindgren, A. (1997). Quantile regression with censored data using generalized $L_{1}$ minimization. Comput. Statist. Data Anal. 23, 509-524.

Nelder, J. A. and Mead, R. (1965). A simplex method for function minimization. Computer J. 7, 308-313.

Neocleous, T. and Portnoy, S. (2009). Partially linear censored quantile regression. Lifetime Data Analysis 15, 357-378.

Parzen, M. I., Wei, L. J. and Ying, Z. (1994). A resampling method based on pivotal estimating functions. Biometrika 81, 341-350.

Peng, L. and Huang, Y. (2008). Survival analysis with quantile regression models. J. Amer. Statist. Assoc. 103, 637-649.

Portnoy, S. (2003). Censored regression quantiles. J. Amer. Statist. Assoc. 98, 1001-1012.

Powell, J. L. (1984). Least absolute deviations estimation for the censored regression. J. Econometrics 25, 303-325.

Qian, J. and Peng, L. (2010). Censored quantile regression with partially functional effects. Biometrika 97, 839-850.

Tsiatis, A. A. (1990). Estimating regression parameters using linear rank tests for censored data. Ann. Statist. 18, 354-372.

Tsimberidou, A. M., O'Brien, S., Khouri, I., Giles, et al. F. G., Kantarjian, H. M., Champlin, R., Wen, S., Do, K-A, Smith, S. C., Lerner, S., Freireich, E. J. and Keating, M. J. (2006). Clinical outcomes and prognostic factors in patients with Richter's syndrome treated with chemotherapy or chemoimmunotherapy with or without stem-cell transplantation. J. Clinical Oncology 24, 2343-2351.

van der Vaart, A. W. and Wellner, J. A. (1996). Weak Convergence and Empirical Processes. Springer, New York.

Wang, H. and Wang, L. (2009). Locally weighted censored quantile regression. J. Amer. Statist. Assoc. 104, 1117-1128.

Wei, L. J., Ying, Z. and Lin, D. Y. (1990). Linear regression analysis of censored survival data based on rank tests. Biometrika 77, 845-851.

Yang, S. (1999). Censored median regression using weighted empirical survival and hazard function. J. Amer. Statist. Assoc. 94, 137-145.

Ying, Z., Jung, S. H. and Wei, L. J. (1995). Survival analysis with median regression models. J. Amer. Statist. Assoc. 90, 178-184.

Yu, K., Lu, Z. and Stander, J. (2003). Quantile regression: Applications and current research areas. J. Roy. Statist. Soc. Ser. D 52, 331-350.

$\mathrm{Yu}$, K. and Jones, M. C. (1998). Local linear quantile regression. J. Amer. Statist. Assoc. 93, 228-237.

Zhang, H. H., Cheng, G. and Liu, Y. (2011). Linear or nonlinear? Automatic structure discovery for partially linear models. J. Amer. Statist. Assoc. 106, 1099-1112.

Department of Statistics and Actuarial Science, The University of Hong Kong, Hong Kong.

E-mail: gyin@hku.hk

Department of Biostatistics, Gillings School of Global Public Health, CB\#7420, The University of North Carolina at Chapel Hill, 27599-7420.

E-mail: dzeng@bios.unc.edu

School of Mathematical Sciences, Beijing Normal University, No. 19, XinJieKouWai St., HaiDian District,Beijing 100875, P. R. China.

E-mail: li hui(abnu.edu.cn

(Received August 2011; accepted April 2013) 